\title{
Forward-Lane Integrity Watchdog System
}

\author{
Adlene Ebenezer, S. Karthik Vignesh, B. Sai Kishore, A. Gokul
}

\begin{abstract}
Today there exist a lot of smart vehicles which can change lane on their own, using their sensors to detect the vehicles around them and using various neural or non-neural algorithms to detect the lane on the road. But these are inherently limited to well-structured road environment and struggle with unstructured road or damaged road. This paper aims to propose a new system, based on cloud and deep-learning neutral networks to process images from each region to train a neural network to be highly efficient in that particular region. We use "Collective wisdom" of people along with data analysis to improve the accuracy of the model.
\end{abstract}

Keywords : convolutional neural network, cloud-computing, data analysis, un-structured roads, collective wisdom

\section{INTRODUCTION}

In the modern era we have data-analytics and neural networks reigning supreme in the field of smart cars; but most smart cars are trained using a limited and specific data set and cannot be deployed in all regions around the world as their neural networks are not trained for those regions due to limited data set. However we do have other methods and techniques to predict the state of the forward lane using other static and predefined algorithms. These algorithms are one time implementation and do not need much service other than being fine-tuned from time-to-time. However trained neural networks have much higher accuracy due to their trainable nature. To accomplish this we need a region specific data and we need a cloud based data-centre and a vehicle fitted with mechanism to communicate with the said cloud and utilize the data provided by it. Cloud is an ondemand computing resource system that's available over a network and can be accessed from anywhere. There are several levels of clouds available; the IAAS (Infrastructure as a service), PAAS (Platform as a service) and SAAS (Software as a service). For our purpose we will be focused on SAAS as we aim to deploy software to train the neural networks. However we also need to be able to collect data and pre-process them, to feed them into the neural network for training them this; involves Big Data analysis to determine which data to use and which to discard. This is also important as it is important to the cloud provider to manage the huge number in-coming data from the users of this technology. Big data is the field concerned with the collection of fast in-coming data and the ability to store them as fast as possible and to process them efficiently.

Revised Manuscript Received on December 30, 2019.

* Correspondence Author

Adlene Ebenezer, Assistant Professor, Department of CSE, SRM Institute of Technology, Ramapuram Tamilnadu, India.

S.Karthik Vignesh, B.Tech. under-graduate, Department of CSE, SRM Institute of Technology, Ramapuram, Tamilnadu, India.

B.Sai Kishore, B.Tech. under-graduate, Department of CSE, SRM Institute of Technology, Ramapuram, Tamilnadu, India.

A.Gokul, B.Tech. under-graduate, Department of CSE, SRM Institute of Technology, Ramapuram, Tamilnadu, India.

(c) The Authors. Published by Blue Eyes Intelligence Engineering and Sciences Publication (BEIESP). This is an open access article under the CC BY-NC-ND license (http://creativecommons.org/licenses/by-nc-nd/4.0/)
We also need to cloud to deploy the updates to vehicles and we use normal TCP/IP connection to accomplish this. The vehicle should have powerful enough computer system and storage to run the neutral network and to interact with the cloud. For this ARM processors can be used. These processors are powerful enough to run neural network and the processors are based on RISC (Reduced Instruction SetComputer) which makes them highly efficient. These can be further paired with high speed solid state storage.The field of smart cars or self-driving cars is vast and is being explored by many mainstream car manufactures denoting the potential and importance of this field. These self-driving cars are defined as vehicles that can navigate from source to destination, while tolerating and taking appropriate action for all conditions encountered on the route. These cars are also called ADS or Automated driving system. However these ADS need a watchdog system to countered the error in the system and to error check their decisions. This necessitates the creation of a watchdog system to monitor the user as well as the ADS vehicle's decisions.Over the years there have been many attempt to automate vehicles and also to detect potholes. There are also paper which is used to guide a vehicle based on structure of the lane as specified in the paper published in IEEE journal where they use stereo-vision[1], Hough transform[1], image segmentation[1] and CNN to predict collision course and to assist in lane among others. Another research uses stereovision along with Dijkstra's algorithm[2] to map the entire road into weighted graphs and the use that to calculate the distance. While its advantageous as no training is required but even this method has a problem as It is largely dependent on image quality and speed of capture. While another paper uses the RGB colour space[3] to calculate the shadow and other details to predicate irregularities in road like potholes. This method also requires no training but has less accuracy than neural network based techniques. Another training-less technique paper uses the stereo vision[4] to re-construct the area and observe it for irregularities. However this technique has a issue of reduced accuracy and more processing time, when travelling at high speed. There is also a paper that uses spatio-temporal saliency[5] to check the environment. It is really close to being perfect as it can still detect in spite of shadows and interferences. However, it requires a good camera as it can not work in low intensity of light. One of the promising paper uses a HCN[6]; a hybrid neural network (a combination of several layers of different neural networks), to train and detect. It can process complex images but is hard to train and takes time and resources to do so.

\section{MODULE DESCRIPTION}

The proposed system consist of four main modules which interact seamlessly with each other to provide the accurate prediction.

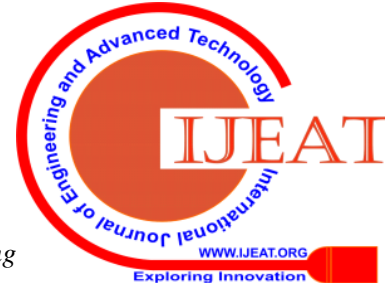




\section{Forward-Lane Integrity Watchdog System}

The first module consist of a camera for imaging purpose, it does not take a continuous video but instead takes several images every second. The second module consist of the cloud network based update and deployment of the trained convolutional neural network. It deals with taking the output of first module, that is, the image and process it to predict its class based on the trained convolutional neural network. The third module consist only of the monitoring system the monitors the prediction percentage and sends it to the car on-board computer system and also intimate the driver of the said vehicle. It monitors the driver's action and registers false positives. It sends the false positives back to the second module; to the cloud. The fourth module is the I/O system and the car on-board computer which is responsible for intimating the driver and also registering his action and reporting it to the monitoring system. The proposed system is split into several modules to provide flexibility to the system; where it would be possible to replace or upgrade only parts of the system as needed.

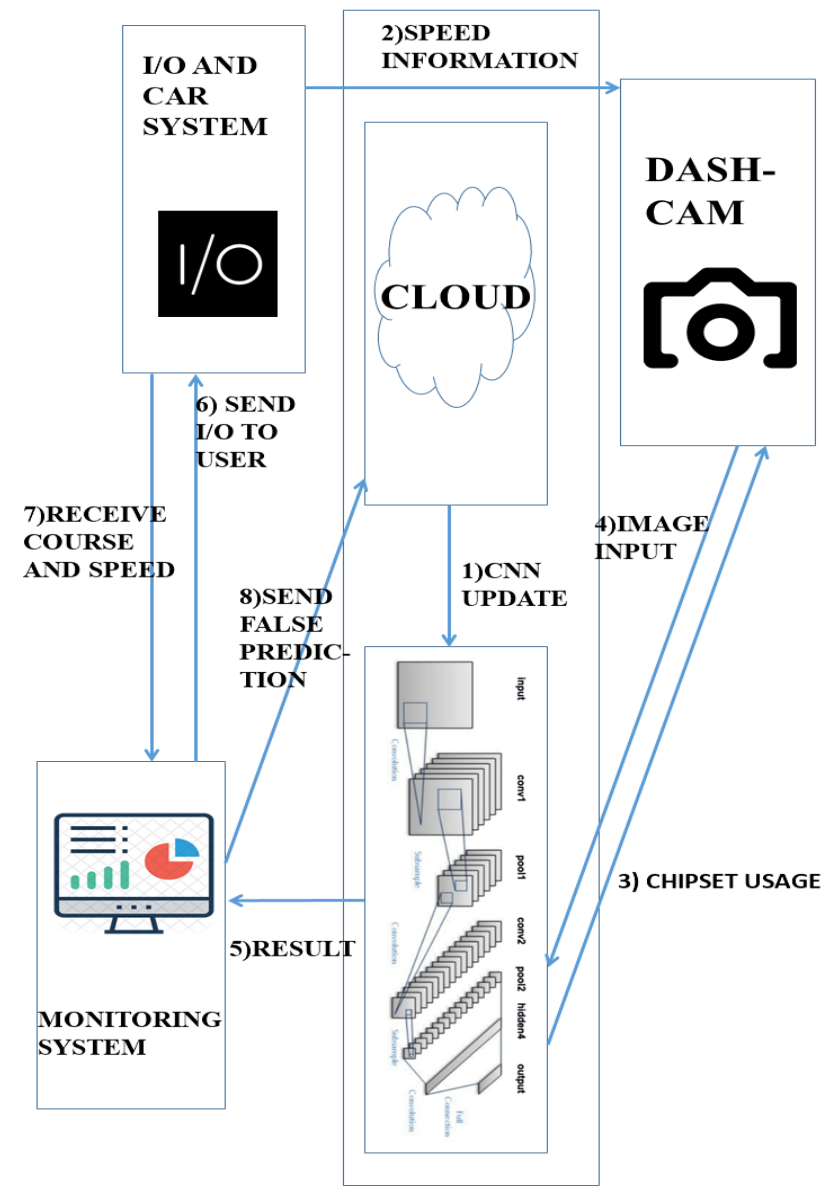

Fig.1 Module Diagram

\section{A. Camera Module}

The camera module is mounted on top of the vehicle to give a better view of the forward lane. How high it is mounted would depend on the range of distance it would need to cover from the vehicle to an arbitrary point on the forward lane. The camera does not take a continuous video like other cameras instead it takes pictures at variable frame rate depending on the speed of the vehicle. This is necessary as not overload the chipset running the neural network while allowing for consistent and accurate prediction. The camera also has night vision as this technology can mainly be a boon in dark night roads. The camera module gets the speed information from the fourth module, the $\mathrm{I} / \mathrm{O}$ and the carsystem module. It also gets the chipset usage of the neural network for assessing how much frames to push to the neural network to process. The ability of the camera itself to push the frames also limits its ability. The produce image is sent to the second module also known as the Deployment module. In the second module it is normalized and processed to feed into neural networks. We use a formula :

\section{$\mathrm{R} 0=(\mathrm{S} / \mathrm{Res})+\mathrm{k}$; if chipset utilization below threshold Where, $\mathrm{S}$ is speed of ADS vehicle,}

Res is Responsiveness (meters after which a frame is taken), $\mathrm{k}$ is the minimum base frame rate

while calculating $\mathrm{R} 0$, the frame rate of the camera we must take $\mathrm{k}$, the base frame rate into consideration as it affects the final resulting frame rate.

\section{B. Deployment Module}

This module contains the Trained CNN and the cloud communication mechanisms to upload data and to update the CNN with a new trained neural network. It is accomplished using standard TCP protocols and IP addressing. The communication has be done by connecting the system through $4 \mathrm{G}$ network or through a home network provided by an ISP. However given the data amount home network from an ISP would be more feasible. The cloud collects data from each region and trains a neural network for that region as an additive to previously existing network for the region. However, in-case of report of large number of wrong prediction from the vehicles of the region would indicate there has been a significant improvement in the region and thus, old data-set would no longer be applicable, so the neural network model for the region is purged. A new neutral neural network is trained form the new data and then updated to the vehicles of that region. It module mainly gets input from the monitoring system with classifiers for the image as 'false positive', 'false negative' and 'positive' along with prediction per cent. The 'positive' are images with low accuracy. The cloud would maintain several virtual machine or VMs for each one of the region. Then the could trains the neural network using standard techniques. The CNN itself only consist of 2 convolutional layer then it is flattened to produce output. The CNN also only consist of 2 classifiers 'Plausible' and 'Failure', where the former means the forward lane integrity is good and the latter meaning the forward lane is damaged and avoid approach. The CNN reports its finding and the corresponding percentage to the monitoring system for evaluation. This module also reports the on-board chipset utilization percentage to the camera system.

\section{Monitoring System Module}

This module gets the prediction from the CNN in the Deployment module and gives the output to the user through the I/O system in the I/O and car system module. It also gets feedback from I/O and car system module as to whether the course was changed or not. 
If course is changed then it compares to see if the result form the CNN is 'plausible' or 'failure'. If it is former then it sends the images with 'false positive' tag if latter then there is no update. If the course did not change and the result is 'plausible' again no action is taken, however is the CNN result is 'failure', then it sends the images with 'false negative' tag to the cloud. It also uses the speed information from the I/O and car system module to advise drastic or nondrastic measure (like full on hard braking or slow braking). It also uploads image that are 'positive' but have low accuracy. The monitoring system gets the information and decides whether the warning is a drastic or non-drastic warning based on the speed of the vehicle given by the formula:

$$
\mathrm{D}_{\text {coeff }}=(\mathrm{CS}+\mathrm{BS} / \mathrm{MS})
$$

Where MS is max speed of vehicle, BS is base speed addition, CS is current speed

When $\mathrm{D}_{\text {coeff }}$ crosses 1 it is understood to be drastic braking or evasion measure. BS is the base speed addition or base speed offset meant to be tweaked and modified for different vehicle based on safety and braking technology and performance. BS is added to CS as an offset.

\section{I/O and Car System Module}

This module is responsible for intimating the user about the result of the network and this system also gets speed information from the vehicle. It sends the speed information to the camera module and the monitoring system module. It has an electronic gyro sensor to sense orientation of the vehicle. Using the speed and change in orientation of the vehicle it can understand the change in direction. If the gyro already indicates arc of a circle like change in car movement then it understands the vehicle is undergoing a turn however any abrupt and sudden change is regard as a change in course and any abrupt change while driving straight is also considered a change in course.

\section{ALGORITHM}

Step 1: Inquire the cloud through a TCP based network for new availability of a new updated version of the CNN. If available download the newly trained CNN from cloud network.

Step 2: The camera module request speed information and chipset utilization information from the I/O and car system module and deployment module respectively. It uses this information to calculate the frame rate R0. It sends the frames to the neural network for evaluation.

$$
\mathrm{R} 0=(\mathrm{S} / \mathrm{Res})+\mathrm{k} \text {; if chipset utilization below threshold }
$$
Where, $\mathrm{S}$ is speed of ADS vehicle,

Res is Responsiveness (meters after which a frame is taken), $\mathrm{k}$ is the minimum base frame rate

Step 3: The deployment module that contains the neural network gets the frame from the camera module and begins processing and outputs with only two classifiers "Plausible" or "Failure" and their predicted percentage is sent to the monitoring system.
Step 4: The monitoring system gets the information and decides whether the warning is a drastic or non-drastic warning based on the speed of the vehicle given by the formula:

$$
\begin{aligned}
& \mathrm{D}_{\text {coeff }}=(\mathrm{CS}+\mathrm{BS} / \mathrm{MS}) \\
& \text { Where MS is max speed of vehicle, } \\
& \text { BS is base speed addition, } \\
& \text { CS is current speed }
\end{aligned}
$$

When $\mathrm{D}_{\text {coeff }}$ crosses 1 it is understood to be drastic braking or evasion measure. BS is the base speed addition or base speed offset meant to be tweaked and modified for different vehicle based on safety and braking technology and performance. BS is added to CS as an offset.

Step 5: If the "Plausible" output has low accuracy associated with it when it comes out of the CNN then it is sent to cloud to add as "Positive" training set.

Step 6: The monitoring system sends the output to the I/O and car system module with drastic or non-drastic or null measure.

Step 7: The I/O and car system module receives the measure form monitoring system and gets the speed information and data from the gyroscope to calculate change in direction of the vehicle. It does this by looking at previous gyroscope data and checks for change in current reading and uses the speed of the vehicle that is acceleration to avoid or etc. to know the change in course. If the gyro already indicates arc of a circle like change in car movement then it understands the vehicle is undergoing a turn however any abrupt and sudden change is regard as a change in course and any abrupt change while driving straight is also considered a change in course.

Step 8: Reports back to monitoring system whether a change in course has taken place or not.

Step 9: If course is changed then it compares to see if the result form the CNN is 'plausible' or 'failure'. If it is former then it sends the images with 'false positive' tag if latter then there is no update. If the course did not change and the result is 'plausible' again no action is taken, however is the $\mathrm{CNN}$ result is 'failure', then it sends the images with 'false negative' tag to the cloud.

Step 10: Repeat process form start.

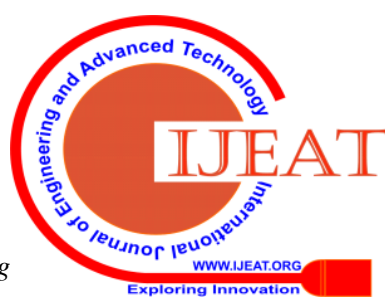




\section{WORKFLOW DIAGRAM}

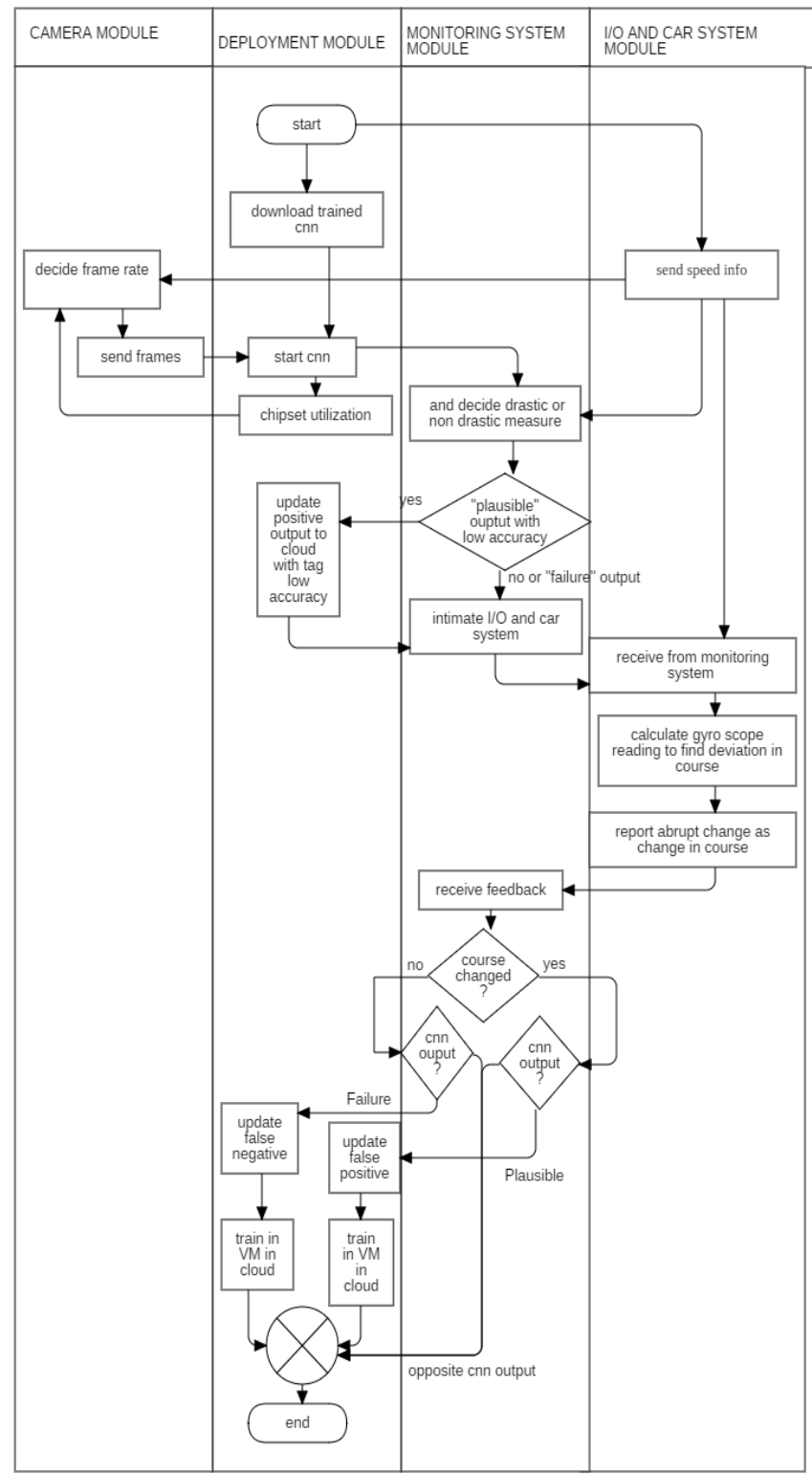

Fig.2 Workflow Diagram

\section{RESULT AND DISCUSSION}

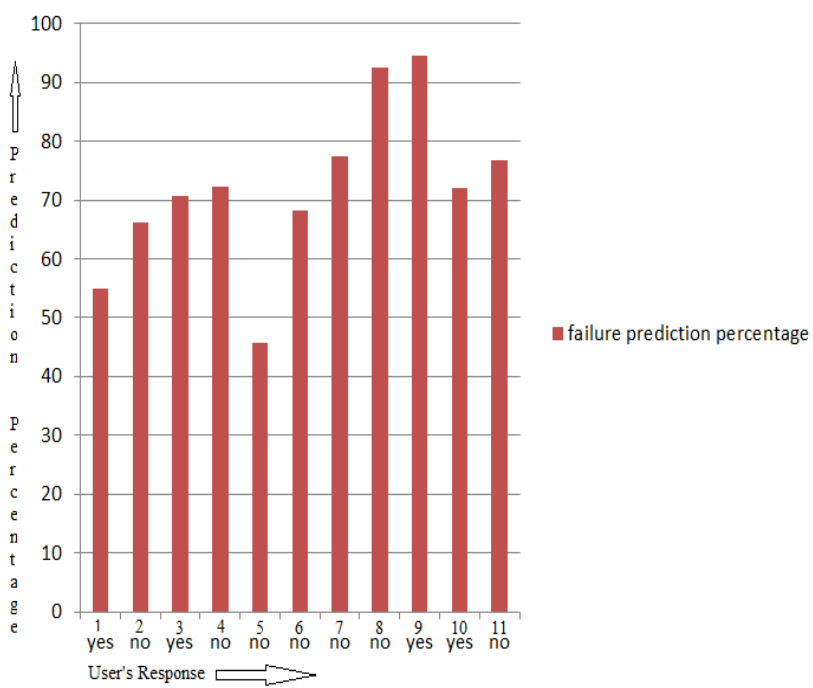

Fig.3 Neutral CNN

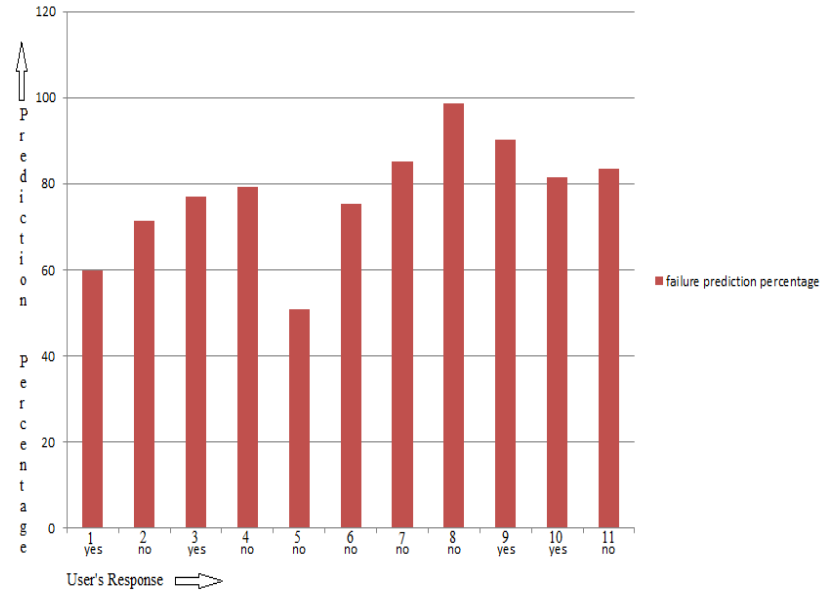

Fig.4 Region Specific CNN

Due to the way the neural network is trained we consider 75 per cent to be the point of determination. Above 75 per cent is considered "failure" and below 75 per cent "plausible". In the neutral CNN also we have 5 differences at slot 2,4,5,6,9 with variations at $13.82 \%, 2.67 \%, 29.31 \%, 6.79 \%, 19.44$ respectively. This gives us 5 failure out of 11 of the scenarios the system was tested in, thus making it highly unreliable.

Here in the region specific trained $\mathrm{CNN}$ it is to be noted that there is a difference in slot 2,3,5,9,10 ( $\mathrm{X}$-axis ). Here the decision of the neural network is opposed to that of the user's (the driver) and it is also to be noted that the variations are huge at 3.5\%, 2.09\%, 24.16\%, $15.27 \%$ and $6.5 \%$ respectively centred and calculated from $75 \%$ as the mark. Out of test of 11 scenario 5 of them are wrong with huge variations making it unreliable, but this has only been account for a single vehicle not a fleet of vehicles. Thus expanding the network of vehicles would improve the accuracy immensely.

When comparing the two networks it is evident that the system with region specific CNN is able to best the neutral CNN in terms of accuracy; as we are able to see that the error range is smaller in region specific CNN than that of the neutral CNN. This accuracy can further increase for the region specific CNN while the same can not be said for the neutral CNN. Thus this supports the claim of the paper to deploy the proposed system to harness the "Collective wisdom" of the people indigenous to the region the system is being deployed to.

\section{CONCLUSION}

This system can be useful for all regions around the world and can be implemented with very low resources and cost. This system can be extended to serve as a basic model for neural network based ADS for regions around world that are specific to the region. The cloud cost would initially be high, but would be reduced as time progresses as the neural network for each region would become more efficient and if we account for adoption rate then even a relatively weak cloud can run this project. The rate then would be constant then decrease as adoption becomes wide-spread. 
However, this project can further be improved using GPS to locate the region where the roads are good and bad. This can help local authorities to repair said damaged road and also possibly warn other users of the system about the road infrastructure in each area. It can also be combined with current mapping service to elevate their performance.

\section{REFERENCE}

1. Wenjie Song, Yi Yang, Mengyin Fu, Yujun Li, Meiling Wang 2018 "Lane Detection and Classification for Forward Collision Warning System Based on Stereo Vision" IEEE, volume: 18, Issue: 12.

2. Yigong Zhang, Yingna Su, Jian Yang, Jean Ponce, Hui Kong 2018 "When Dijkstra Meets Vanishing Point: A Stereo Vision Approach for Road Detection" IEEE, Volume: 27, Issue: 5.

3. Amila Akagic, Emir Buza, Samir Omanovic 2017 "Pothole Detection: An Efficient Vision Based Method Using RGB Color Space Image Segmentation" IEEE, $40^{\text {th }}$ MIPRO.

4. Muhammad Uzair Ul Haq, Moeez Ashfaque, Senthan Mathavan, Khurram Kamal, Adeel Ahmed 2019 "Stereo-Based 3D Reconstruction of Potholes by a Hybrid, Dense Matching Scheme" IEEE, Volume: 19, Issue:10.

5. Dong-Won Jang, Rae-Hong Park 2016 "Pothole detection using spatio-temporal saliency" IEEE, Volume: 10, Issue: 9.

6. Ye Li, Lili Guo, Jun Rao, Lele Xu, Shan Jin 2019 "Road Segmentation Based on Hybrid Convolutional Network for HighResolution Visible Remote Sensing image" IEEE, Volume: 16, Issue: 4.

\section{AUTHORS PROFILE}

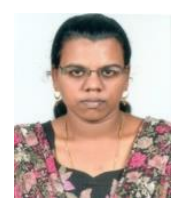

Adlene Ebenezer, Assistant professor, Department of CSE, SRM Institute of technology, Ramapuram Tamilnadu, India.

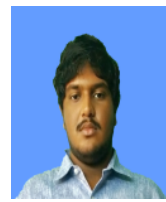

S.Karthik vignesh, B.Tech. under-graduate, Department of CSE, SRM Institute of technology, Ramapuram, Tamilnadu, India.

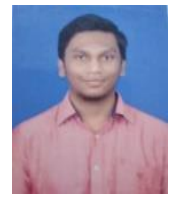

B.Sai Kishore, B.Tech. under-graduate, Department of CSE, SRM Institute of technology, Ramapuram, Tamilnadu, India.

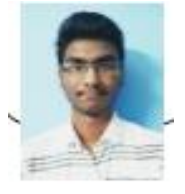

A.Gokul, B.Tech. under-graduate, Department of CSE, SRM Institute of technology, Ramapuram, Tamilnadu, India. 